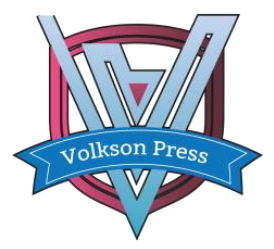

Contents List available at VOLKSON PRESS

Mechanical and Control Engineering (MCE)

DOI : http://doi.org/10.26480/wsmce.01.2017.127.128

\title{
AN ANALYSIS ON DIAGNOSTIC METHOD OF ROLLER BEARING FAULTS BASED ON AUDITORY SPECTRUM AND SPECTRAL CORRELATION
}

\author{
Li Yungong*, Xu Jinfang, Da Li, Zhang Qilin \\ School of Mechanical Engineering and Automation, Northeastern University, Shenyang 110004, China \\ *Corresponding Author Email: ygli@mail.neu.edu.cn
}

This is an open access article distributed under the Creative Commons Attribution License, which permits unrestricted use, distribution, and reproduction in any medium, provided the original work is properly cited

\section{ARTICLE DETAILS}

\section{Article History:}

Received 02 october 2017 Accepted 06 october 2017

Available online 11 november 2017

Keywords

EA model, characteristic auditory spectrums, spectral correlation, fault diagnosis

\section{ABSTRACT}

Human auditory system possesses a desirable capacity for analyzing, processing and identifying signals. Whereas different types of rolling bearing faults cause different vibration noises, a new method based on early auditory model (EA model) is proposed for the diagnosis of rolling bearing faults. Firstly, according to characteristics of mechanical vibration signal, the EA model was established by simulating the human auditory system. Secondly, sample signals were processed by the EA model to build sets of auditory spectrums, the characteristics of which were extracted with the purpose of obtaining the characteristic auditory spectrums that reflected the overall characteristics of failure states, so as to further simplify the data. At last, based on spectral correlation, it applied integrated correlation coefficient to identify fault type. Experimental results show that the method is capable of extracting and distinguishing the overall characteristics of 12 types of rolling bearing fault states (four different degrees of faults and normal state of inner race, outer race and rolling element) with almost 100 percent accuracy possesses considerable feasibility and certain potentialities in practical application.

\section{Introduction}

Rolling bearings are widely used in rotating machineries, so that their states directly affect the performance of machinery equipment. In order to reduce the accidents of rotating machinery, it is necessary to realize accurate diagnosis of rolling bearing faults. At present, there are three types of characteristic extraction methods for rolling bearing faults i.e. spectrum analysis method, envelope analysis method and shock pulse method [1-4].

At the work site, when different types of rolling bearings faults occur, it will produce different sounds during the process of rotating. As a result of that vibration signals and noise signals are homologous, it is able to conduct characteristic extraction and fault identification to vibration signals of bearings through the processing mechanism of human auditory system. With respect to auditory system simulation, the current auditory model in the field of auditory periphery system simulation is already mature, and its performance is comparatively close to biological experiments [5].

In consideration of advantages of auditory model and the conditions of diagnosis of rolling bearing faults, this paper will introduce EA model into diagnosis of rolling bearing faults, and also design the method of fault characteristics extraction and identification in line with multiple auditory spectrum. By utilizing the data validation for bearing vibration provided by the American Case Western Reserve University, it indicates that the proposed method possesses relatively high accuracy in diagnosis of roller bearing faults.

\section{METHOD OVERVIEW}

Implementation process of the proposed method is shown in Figure 1.

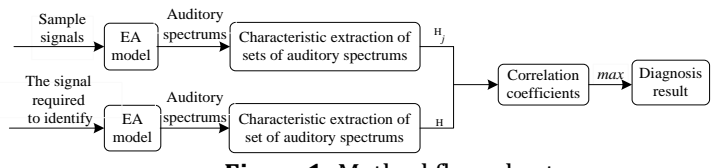

Figure1: Method flow chart
EA mode's implementation process is shown in Figure 2.

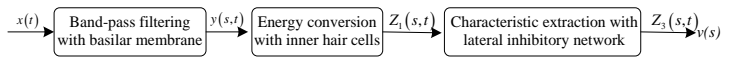

Figure 2: EA model flow chart

In order to simulate band-pass filtering of the basilar membrane, Gammatone filter bank is applied into EA model to conduct frequency decomposition.

The number of filters is $n$ that is set to 150 , and $h(s, t)$ represents the $s$ th filter, where center frequency is determined by $s$. The response expression of basilar membrane model to signal $x(t)$ is given by:

$$
y(s, t)=x(s, t){ }_{t} h(s, t)
$$

where $y(s, t)$ denotes the output of the basilar membrane and $*_{t}$ denotes the convolution of the time domain.

Let $f_{s}$ be center frequency of the $s$-th filter and $f_{s}$ is uniformly distributed the expression of GT filter is written as:

$h(s, t)=B^{m-1} t^{m-1} e^{-2 \pi B t} \cos \left(2 \pi f_{s} t+\varphi\right)$

where $m$ is the order of the filter. $m$ is 4 and $\varphi$ is equal to 0 normally. $B$ is calculated by:

$B=1.019 \times E R B\left(f_{s}\right)=1.019 \times\left(24.7+0.108 f_{s}\right)$

where $\operatorname{ERB}\left(f_{s}\right)$ denotes the equivalent rectangular band width of the filter at the center frequency $f_{s}$.

The energy conversion expression of inner hair cell model is written as: $Z_{1}(s, t)=g\left[\frac{\partial y(s, t)}{\partial t}\right] *{ }_{t} \alpha(\mathrm{t})$

\subsection{EA model}


Where $\alpha(t)$ denotes a low-pass filter.

$g(*)$ as a Sigmoidal function, is introduced into the process of energy conversion in EA mode:

$$
g\left(\frac{\partial y(s, t)}{\partial t}\right)=\frac{1}{1+\exp \left[-\gamma \times \frac{\partial y(s, t)}{\partial t}\right]^{-\frac{1}{2}}}
$$

where $\gamma$ denotes the coefficient of nonlinear amplitude compression, and is set to 2 in this study.

Lateral inhibitory network of EA model mainly conducts filtering and halfwave rectification to the signals.

$$
\begin{aligned}
& Z_{2}(s, t)=\frac{\partial Z_{1}(s, t)}{\partial s} *_{s} \beta(s) \\
& Z_{3}(s, t)=\max \left(Z_{2}(s, t), 0\right)
\end{aligned}
$$

Where $\beta(s)$ denotes a low-pass filter.

Auditory spectrum without time parameter expression's is written as:

$$
v(s)=\frac{1}{T} \sum_{0}^{T} Z_{3}(s, t)
$$

\subsection{Characteristic Auditory Spectrums}

Let $J$ be the number of states, and each state contains $K$ segments sample signals. $v_{j}{ }^{k}(s)$ denotes the auditory spectrum of the $k$-th segment in the $j$-th state, and the auditory spectrum set in the $j$-th state is given by:

$$
\mathbf{V}_{j}=\left[v_{j}{ }^{1}(s), v_{j}{ }^{2}(s), \mathrm{L}, v_{j}{ }^{k}(s)\right]^{\prime}
$$

Characteristic auditory spectrums are extracted, the average auditory spectrum $\psi_{j}(s)=\frac{1}{K} \sum_{k=1}^{K} v_{j}{ }^{k}(s)$, the minimum auditory spectrum $\varphi_{j}(s)=\max \left\{v_{j}{ }^{k}(s)\right\}$, the maximum auditory spectrum $\chi_{j}(s)=\min \left\{v_{j}{ }^{k}(s)\right\}$ in the $j$-th state.
The characteristic auditory spectrums in the $j$-th state is denoted by the Matrix $\mathbf{H}_{j}$, which is written as:

$$
\mathbf{H}_{j}=\left\lfloor\psi_{j}(s) \quad \varphi_{j}(s) \quad \chi_{j}(s)\right\rfloor^{\prime}
$$

\subsubsection{Identification Method}

By applying mathematical expression, it identifies that integrated correlation coefficient between the signal required to identify and the $\ell$ th state has the maximum value. It is noted that the signal required to identify is closest to the $\ell$-th state, which means it belongs to the $\ell$-th sample state.

\section{CONCLUSION}

It proposed a method of diagnosis for rolling bearing faults, and also designed a post-process method of auditory spectrum and a fault identification method. Obvious distinctions exist in sets of auditory spectrums and characteristic auditory spectrum corresponding to the 12 states of roller bearing, while type of spectrum is stable, which is suitable to be the fault characteristics for roller bearing. In consideration of that auditory spectrum reflects the condition of perception of the auditory system to signals, it is feasible to regard the auditory spectrum as fault characteristics to detect mechanical faults with vibration and noise.

\section{REFERENCES}

[1] Wen, C., Dong, L., Jin, X. 2015. Feature extraction of bearing vibration signals using second generation wavelet and spline-based local mean decomposition. Journal of Shanghai Jiaotong University (Science), 20 (1), $56-60$.

[2] Tabrizi, A., Garibaldi, L., Fasana, A.B. 2015. Early damage detection of roller bearings using wavelet packet decomposition, ensemble empirical mode decomposition and support vector machine. Meccanica, 50 (3), 865874.

[3] Yao, J., Tang, B., Zhao, J. 2016. A Fault Feature Extraction Method for Rolling Bearing Based on Pulse Adaptive Time-Frequency Transform. Shock and Vibration, 2 (1), 1-12.

[4] Ou, L., Yu, D., Yang, H. 2016. A new rolling bearing fault diagnosis method based on GFT impulse component extraction. Mechanical Systems and Signal Processing, 81 (2), 162-182.

[5] Yuanfeng, M.A., Ke'an, C., Na, W.B. 2009. Application of auditory spectrum-based features into acoustic target recognition. Acta Acustica, $34(2), 142-150$ 